\title{
Influência da caminhada na cognição e composição corporal de mulheres idosas
}

\section{Influence of walking on cognition and body composition of older women}

\section{AUTORES \\ Rafael Afonso de Oliveira ${ }^{1}$ (ID) \\ Vinícius Nagy Soares ${ }^{2}$ (i) \\ Ricardo Aurélio Carvalho Sampaio ${ }^{3}$ (D) \\ Paula Teixeira Fernandes ${ }^{4}$ (D) \\ 1 Universidade Estadual de Campinas, Faculdade de Educação Física, Grupo de Estudos em Psicologia do Esporte e Neurociências. Campinas, São Paulo, Brasil. \\ 2 Universidade Estadual de Campinas, Programa de Pós-graduação em Gerontologia, Faculdade de Ciências Médicas, Grupo de Estudos em Psicologia do Esporte Neurocîncias. Campinas, São Pado, Brasil. \\ 3 Universidade Federal de Sergipe, Departamento de Educação Física, São Cristóvão, Sergipe, Brasil. \\ 4 Universidade Estadual de Campinas, \\ Departamento de Ciências do Esporte, Faculdade \\ de Educacão Física e Programa de Pós-graduação \\ em Gerontologia, Faculdade de Ciências Médicas. \\ Grupo de Estudos em Psicologia do Esporte e \\ Neurociências. Campinas, São Paulo, Brasil.}

\section{CONTATO}

Paula Teixeira Fernandes

paula@fef.unicamp.br

Av. Érico Veríssimo, 701, Cidade Universitária

Zeferino Vaz, Campinas, São Paulo, Brasil.

CEP: 13083-851.

DOI

$10.12820 /$ rbafs. $24 \mathrm{e} 0081$

\begin{abstract}
RESUMO
O objetivo deste estudo foi verificar a influência da caminhada em variáveis cognitivas e antropométricas de mulheres idosas. Foram recrutadas 30 mulheres destreinadas, sem doenças neurodegenerativas, com média de idade de $64,40 \pm 3,71$ anos, divididas por conveniência em grupo de intervenção $(n=16)$ e controle $(n=14)$. O grupo de intervenção foi submetido à prática de jogos desportivos, caminhada e alongamento, em ambientes abertos, durante três meses, duas vezes por semana. $\mathrm{O}$ grupo controle não participou de nenhum programa de atividade física. Antes e após a intervenção, todas as idosas foram submetidas aos testes Códigos WAIS-III, Color Trail Test (CTT-A e CTT-B), e medidas de massa corporal e estatura (determinação do índice de massa corporal - IMC), circunferência da cintura e do quadril (razão cintura-quadril - RCQ). O grupo de intervenção melhorou o desempenho no teste Códigos WAIS-III ( $\Delta=8,68$; IC95\%: 6,7; 10,7) e reduziu os tempos de execução dos testes CTT-A ( $\Delta=-7,94$; IC95\%: -13,1; -2,8) e CTT-B ( $\Delta=-18,59$; IC95\%: -31,4; -5,8). As modificações do grupo controle não foram estatisticamente significativas ( $p>0,05)$. Conclui-se que a prática de caminhada pode melhorar habilidades cognitivas de mulheres idosas destreinadas.
\end{abstract}

Palavras-chave: Atividade física; Cognição; Envelhecimento; Psicologia do esporte.

\section{ABSTRACT}

The aim of this study was to verify the influence of walking on cognitive and anthropometric variables of older women. Thirty untrained women without neurodegenerative diseases were recruited, with mean age of $64.40 \pm 3.71$ years, divided for convenience in intervention $(n=16)$ and control $(n=14)$ groups. The intervention group played outdoor activities, such as sports, walking, and stretching for three months, twice a week. The control group was not engaged in any physical activity program. All volunteers were submitted to the Codes WAIS-III, Color Trail Test (CTT-A and CTT-B), Body Mass, Body Mass Index (BMI) and Waist-Hip Ratio (WHR) tests at baseline and after the study period. The intervention group improved performance of the Codes WAIS-III $(\Delta=8.68$; CI95\%: 6.7; 10.7) and reduced the execution time of CTT-A $(\Delta=-7.94 ; C I 95 \%:-13.1 ;-2.8)$ and $C T T-B(\Delta=-18.59 ; C I 95 \%: 31.4 ;-5.8)$. Changes in the control group were not statistically significant $(p>0,05)$. We showed that walking improves cognitive abilities of untrained elderly women.

Keywords: Aging; Cognition; Physical activity; Sport psychology.

\section{(cc) BY-NC-SA}

Este obra está licenciado com uma Licença

Creative Commons Atribuição-NãoComercial-

CompartilhaIgual 4.0 Internacional.

\section{Introdução}

O estudo Fragilidade em Idosos Brasileiros (FIBRA) revela que 1 a cada 4 residentes da comunidade apresenta declínio cognitivo, sendo a população feminina a mais acometida ${ }^{1}$. No município de Bagé, Rio Grande do Sul, os resultados foram ainda mais alarmantes, nos quais as estimativas de prevalência de déficit cognitivo apontam para 1 a cada 3 idosos ${ }^{2}$. Os primeiros indícios de declínio cognitivo são as dificuldades no armazena- mento e/ou recordação de informações recentes, mas sem comprometimento nas atividades do cotidiano ${ }^{3}$. Durante o processo de envelhecimento, além da memória, outras habilidades podem entrar em declínio como a atenção, velocidade de processamento da informação e funções executivas ${ }^{3}$.

Tais perdas cognitivas podem levar à demência, comprometendo a independência e a autonomia do idoso. Os principais fatores de risco são o aumento da idade, 
baixa escolaridade ${ }^{4}$, carências alimentares, hiperinsulinemia $^{5}$, doenças cardiovasculares ${ }^{6}$ e sedentarismo ${ }^{7}$. A obesidade é outro potencial fator de risco que deve ser considerado, tendo em vista a sua elevada prevalência. Em pessoas de meia idade, $24,4 \%$ das mulheres apresentam obesidade, prevalência ligeiramente superior em comparação aos homens $(16,8 \%)^{8}$. Especialmente em idosos, a obesidade provoca redução da mobilidade nos espaços de vida ${ }^{9}$, o que pode mediar aspectos sociais, como o baixo envolvimento social, agravando os fatores de risco associados ao declínio cognitivo.

Por outro lado, estudos revelaram que a prática regular de exercícios físicos estimula fatores neurotróficos ${ }^{7}$, potencializa mecanismos anti-inflamató$\operatorname{rios}^{10}$ e diminui o risco cardiovascular ${ }^{6}$. Apesar disso, a intensidade do esforço físico mais indicada à função cognitiva ainda é um assunto controverso na literatura. Considerando os efeitos agudos do exercício físico, mudanças mais significativas foram observadas em altas intensidades, especialmente em relação ao BDNF (Brain Derived Neurotrophic Factor) ${ }^{11}$. No entanto, uma metanálise verificou que exercícios de baixa intensidade apresentaram efeitos positivos na cognição de idosos, imediatamente após o exercício e considerando um intervalo pós-exercício ${ }^{12}$. Ainda que os efeitos de mudança tenham sido baixos, os autores verificaram que fatores como duração e intensidade do exercício, tipo de avaliação cognitiva e nível de atividade física são importantes variáveis mediadoras ${ }^{12}$.

Em relação às adaptações crônicas, idosos com declínio cognitivo moderado que praticaram atividade aeróbica progressiva (i.e., caminhada) apresentaram melhores resultados cognitivos globais em comparação ao grupo controle ${ }^{13}$. Uma metanálise envolvendo 42 estudos revelou que a caminhada praticada ao ar livre auxilia na diminuição da pressão arterial, da gordura corporal, do índice de massa corporal (IMC), dos sintomas depressivos, entre outros benefícios ${ }^{14}$.

Apesar da relação entre exercício e desempenho cognitivo ser discutida há certo tempo na literatura, o corpo de informações ainda é discrepante em relação ao tipo de atividade física efetiva à população longeva. Diante desse cenário, buscou-se verificar a influência da caminhada em variáveis cognitivas e antropométricas de mulheres idosas.

\section{Métodos}

Este é um estudo quase-experimental, que inicialmente recrutou 31 mulheres idosas, destreinadas e residentes na cidade de Jaguariúna, São Paulo, Brasil. Os critérios de inclusão para seleção da amostra foram: 1) residir na comunidade; 2) $\geq 60$ anos de idade; 3) autodeclarar-se apta à prática de atividade física; 4) não praticar exercícios físicos sistematizados há 3 meses ou mais; e 5) não apresentar doenças neurodegenerativas (autorrelato). Foram excluídas aquelas que declararam doenças cardiovasculares ou pulmonares sem acompanhamento médico (condições não controladas). Toda a pesquisa ocorreu em parceria com o programa "ProAtiv", vinculado à Secretaria de Juventude, Esporte e Lazer da Prefeitura Municipal de Jaguariúna, São Paulo. O programa possui um ciclo anual de atividades e um período de recesso de três meses, período este designado ao cadastro de pessoas interessadas em praticar atividade física. Após o recesso, compareceu-se ao local designado ao início das atividades, sendo apresentado o estudo, informando a importância, os objetivos, o protocolo de atividade física e os aspectos éticos. As participantes que concordaram assinaram o Termo de Consentimento Livre e Esclarecido (TCLE) e participaram da coleta de dados. Não foi realizado cálculo amostral a priori, sendo o tamanho da amostra definido por conveniência.

As voluntárias foram alocadas em grupo de intervenção ou controle, conforme o interesse pessoal na prática de um programa de atividade física. Dezessete voluntárias declararam interesse em participar do grupo de intervenção, enquanto que as demais formaram o grupo controle (i.e., não praticaram o programa de atividade física). Todas as voluntárias foram submetidas às mesmas avaliações, uma semana antes (pré) e uma semana depois (pós) da intervenção. As avaliações foram realizadas pelo mesmo avaliador, que não sabia quem era do grupo de intervenção ou controle. Para a aplicação dos testes cognitivos, o avaliador passou por um treinamento com um profissional credenciado no Conselho Regional de Psicologia do Estado de São Paulo (CRP-SP). As avaliações foram realizadas no mesmo ambiente de prática das atividades físicas (procurou-se local tranquilo, com o mínimo de distrações), individualmente e em horários agendados, em mesas e cadeiras do parque. As participantes foram instruídas a manterem suas rotinas habituais, não existindo interferência ou controle sobre hábitos alimentares e atividades da vida diária.

Para a avaliação antropométrica, foram medidas a massa corporal e a estatura por meio de balança mecânica (Welmy $110 \mathrm{CH}$, São Paulo, Brasil). As participantes mantiveram-se descalças, com os braços estendi- 
dos e relaxados. A circunferência de cintura foi avaliada com fita métrica, posicionada entre o ponto médio do rebordo costal inferior e a crista ilíaca. Para a avaliação da circunferência de quadril, a fita métrica foi posicionada na região de maior amplitude do glúteo ${ }^{15}$. Para fins de análise, cada medida antropométrica foi executada uma única vez. O índice de massa corporal (IMC) e a relação cintura-quadril (RCQ) foram adotados os seguintes pontos de corte: $\mathrm{IMC}=$ peso $(\mathrm{kg}) /$ altura $^{2}$ (m); RCQ = perímetro da cintura $(\mathrm{cm}) /$ perímetro do quadril $(\mathrm{cm})$. Foram adotadas classificações nos seguintes pontos de corte: IMC: magreza: $<22 \mathrm{~kg} / \mathrm{m}^{2} ; 22-27$ $\mathrm{kg} / \mathrm{m}^{2}=$ peso normal; $>27 \mathrm{~kg} / \mathrm{m}^{2}=$ sobrepeso $^{16}$; RCQ: $<0,76=$ baixo; $0,76-0,83=$ moderado $>0,83=$ alto $^{17}$.

$\mathrm{Na}$ avaliação cognitiva, foi aplicado o Subteste Códigos da Escala Wechsler de Inteligência (Códigos WAIS-III) para verificar a Atenção Seletiva e a Velocidade de Processamento da Informação ${ }^{18}$. O instrumento consiste em símbolos associados a números apresentados na parte superior da ficha de respostas. Abaixo existem números aleatórios contendo lacunas para o preenchimento dos símbolos correspondentes, que devem ser preenchidos o mais rápido possível. A duração do teste é de 120 segundos, sendo a pontuação determinada pela quantidade de símbolos desenhados corretamente neste período de tempo pré-determinado. Esse instrumento foi adaptado e validado para a população brasileira, podendo ser aplicado a pessoas de 16 a 89 anos $^{18}$. Em seguida, foi aplicado o Color Trail Test (CTT) para avaliar funções executivas ${ }^{19}$. Esse teste consiste em dois exercícios de ligações de círculos numerados de 1 a 25. No exercício A (CTT-A), as participantes realizaram ligações de 1 a 25 , na ordem direta. No exercício B (CTT-B), a ligação de 1 a 25 foi repetida, porém as participantes deveriam alternar entre números e letras. Ambos os testes foram precedidos por treinamento e o escore foi igual ao tempo de execução de cada tarefa. Assim, quanto maior o escore, pior o desempenho cognitivo. Ambos os testes (Códigos WAIS-III e CTT) foram validados para uso em amostras brasileiras ${ }^{20,21}$.

O programa de intervenção foi ministrado por profissionais de Educação Física, durante 3 meses, ao ar livre, no período matutino, em parques arborizados da cidade de Jaguariúna, São Paulo. As sessões foram coletivas, com frequência de duas vezes por semana e duração de 60 minutos.

Nos 5 minutos iniciais, eram realizados exercícios de alongamento dos músculos do pescoço, membros superiores, inferiores, dorsais e peitorais, solicitando às voluntárias que se mantivessem em posição estática durante 20 segundos. Nos 10 minutos seguintes, foram realizadas atividades lúdicas e jogos pré-desportivos com intuito de criar um ambiente favorável à pratica de atividade física, sendo este agradável, integrativo e socializador. Nesta etapa, as atividades eram modificadas a cada sessão, mas com objetivos e intensidades similares, sem afetar o volume e a intensidade da sessão. $\mathrm{Na}$ sequência, as participantes caminhavam em diferentes planos como subidas, descidas, solos regulares e irregulares, com duração de 30 a 40 minutos. O percurso era modificado a cada sessão de treino, visando a variação de ambientes e, consequentemente, de estímulos sensoriais. Sobre a intensidade, apesar de não haver controle individual da percepção subjetiva do esforço, as participantes foram instruídas a caminharem em um ritmo moderado, não muito leve, mas também que não causasse dor ou desconforto. Os 5 minutos finais eram dedicados aos exercícios de relaxamento, como técnicas de respiração e de alongamento estático.

A análise descritiva foi adotada para caracterizar as variáveis antropométricas e cognitivas. Os testes de Shapiro-Wilk e de Levene foram utilizados para a verificação da normalidade e homogeneidade dos dados, respectivamente. $\mathrm{Na}$ linha de base, os testes t para amostras independentes ou Mann-Whitney foram usados para comparar as variáveis contínuas entre os grupos, enquanto que o teste Exato de Fisher foi usado para comparar proporções de variáveis categóricas entre os grupos. Para verificar o efeito da intervenção foram realizados modelos mistos, tendo como variáveis dependentes a massa corporal, IMC, RCQ, códigos WAIS-III, CTT-A e CTT-B. Como fatores fixos, foram considerados o grupo (intervenção e controle) e o tempo (pré e pós). As matrizes de covariância foram definidas por AIC (Akaike Information Criterion) e o tamanho do efeito das diferenças entre os grupos foi estimado por d de Cohen (pontos de corte $^{22}: \leq 0,19$ = "insignificante"; $0,20-0,49$ = "pequeno"; 0,50-0,79 = "médio"; $0,80-1,29$ = "grande"; 1,30 ou superior = "muito grande"). O nível de significância estatística adotado foi de $5 \%$ e todas as análises foram feitas no Statistical Package for the Social Science (SPSS; IBM, Chicago, IL, EUA), versão 25,0.

$\mathrm{O}$ estudo seguiu as recomendações e requisitos da Resolução 466/12 do Conselho Nacional de Saúde e suas complementares, com aprovação do Comitê de Ética em Pesquisa da Universidade Es- 
tadual de Campinas (parecer $\mathrm{n}^{\circ} 821.784$ e CAAE $\mathrm{n}^{\circ}$ 34386514.4.0000.5404).

\section{Resultados}

Trinta e uma voluntárias foram convidadas a participar do estudo, não sendo registrada nenhuma recusa. Em média, a amostra apresentou 64,40 $\pm 3,72$ anos de idade, massa corporal de 68,67 $\pm 10,16 \mathrm{~kg}$, IMC de 28,69 $\pm 4,41 \mathrm{~kg} / \mathrm{m}^{2}$ e RCQ de 0,90 $\pm 0,07$. Em relação ao desempenho cognitivo inicial, as médias foram de 28,37

Tabela 1 - Comparação na linha base entre os grupos intervenção e controle, em mulheres idosas do município de Jaguariúna, São Paulo.

\begin{tabular}{lccccc}
\hline \multirow{2}{*}{ Variáveis } & \multicolumn{2}{c}{$\begin{array}{c}\text { Intervenção } \\
(\mathrm{n}=16)\end{array}$} & \multicolumn{2}{c}{$\begin{array}{c}\text { Controle } \\
(\mathrm{n}=14)\end{array}$} & $\begin{array}{c}\text { P } \\
\text { valor }\end{array}$ \\
\cline { 2 - 5 } & Média & $(\mathrm{DP})$ & Média & $(\mathrm{DP})$ & \\
\hline Idade & 64,06 & $(4,42)$ & 64,79 & $(2,83)$ & 0,257 \\
Antropométricas & & & & & \\
$\quad$ Massa corporal (kg) & 66,94 & $(8,34)$ & 70,63 & $(11,92)$ & 0,329 \\
IMC $\left(\mathrm{kg} / \mathrm{m}^{2}\right)$ & 27,19 & $(2,71)$ & 30,39 & $(5,39)$ & 0,059 \\
RCQ & 0,88 & $(0,06)$ & 0,92 & $(0,07)$ & 0,073 \\
Cognitivas & & & & & \\
Códigos WAIS-III & 32,68 & $(15,08)$ & 23,43 & $(5,55)$ & 0,093 \\
CTT-A & 83,25 & $(42,68)$ & 83,90 & $(39,77)$ & 0,953 \\
CTT-B & 135,33 & $(59,14)$ & 133,50 & $(9,57)$ & 0,960 \\
Classificação IMC & $\mathrm{n}$ & $(\%)$ & $\mathrm{n}$ & $(\%)$ & 0,185 \\
Magreza & 0 & $(0,00)$ & 1 & $(7,14)$ & \\
Peso normal & 8 & $(50,00)$ & 3 & $(21,43)$ & \\
Sobrepeso & 8 & $(50,00)$ & 10 & $(71,43)$ & \\
Risco cardiovascular & & & & & 0,567 \\
Moderado & 3 & $(18,80)$ & 2 & $(14,30)$ & \\
Alto & 13 & $(81,20)$ & 12 & $(85,70)$ & \\
\hline
\end{tabular}

$\mathrm{IMC}=$ índice de massa corporal; $\mathrm{RCQ}=$ relação cintura quadril; Códigos WAIS-III = Subteste Códigos da Escala Wechsler de Inteligência; $\mathrm{CTT}=$ Color Trail Test; $\mathrm{DP}$ = desvio padrão. $\pm 12,39$ no teste Códigos WAIS III, 95,81 \pm 43,12 no teste CTT-A e 134,60 $\pm 44,44$ no teste CTT-B. Durante o estudo, uma voluntária desistiu por motivos pessoais, sem qualquer relação com o protocolo de intervenção. Não houve relato de lesão ou desconforto e todas as participantes submetidas à intervenção alcançaram frequência mínima de $75 \%$, participando de pelo menos 18 sessões. Na linha de base, não houve diferenças estatisticamente significativas entre os grupos para as variáveis antropométricas e cognitivas (Tabela 1).

Os resultados do modelo misto estão na Tabela 2 . Houve efeito do tempo $[\mathrm{F}(1,28)=35,45]$ e da interação $[\mathrm{F}(1,28)=40,44]$ no teste Códigos WAIS-III, indicando que o grupo que praticou atividade física melhorou o desempenho cognitivo, sendo o tamanho do efeito muito grande $(\mathrm{d}=2,18)$. Apesar de ter sido encontrado efeito do tempo $[\mathrm{F}(1,20,05)=6,89]$ no CTT-A, os intervalos de confiança indicaram que somente o grupo de intervenção diminuiu o tempo de conclusão do teste $(\mathrm{d}=0,58)$. Resultado similar foi encontrado no CTT-B, no qual houve efeito da interação $[F(1,8,62)=5,50)$, cujos intervalos de confiança demonstraram que o grupo de intervenção apresentou variação negativa do escore médio $(\mathrm{d}=1,20)$. Em relação às variáveis antropométricas, não foram encontradas variações estatisticamente significativas após a intervenção, em ambos os grupos.

Ao término do estudo, 10 (62,5\%) voluntárias do grupo de intervenção estavam com sobrepeso e $6(37,5 \%)$ com peso normal. No grupo controle, 11 $(78,6 \%)$ apresentaram sobrepeso, $2(14,3 \%)$ peso normal e $1(7,1 \%)$ baixo peso, sem diferenças estatisticamente significativas entre os grupos $(\mathrm{p}=0,226)$. Sobre o risco cardiovascular, 2 (12,5\%) voluntárias do grupo de intervenção foram classificadas com baixo risco, 4

Tabela 2 - Comparação das variáveis antropométricas e cognitivas entre os grupos intervenção e controle, pré e pós 12 semanas de caminhada, em mulheres idosas do município de Jaguariúna, São Paulo.

\begin{tabular}{|c|c|c|c|c|c|c|}
\hline \multirow{2}{*}{ Variáveis } & \multicolumn{2}{|c|}{ Intervenção $(\mathrm{n}=16)$} & \multicolumn{2}{|c|}{ Controle $(n=14)$} & \multirow{2}{*}{ P tempo } & \multirow{2}{*}{ P interação } \\
\hline & $\Delta$ & (IC95\%) & $\Delta$ & (IC95\%) & & \\
\hline \multicolumn{7}{|l|}{ Antropométricas } \\
\hline Massa corporal $(\mathrm{kg})$ & 0,01 & $(-7,3 ; 7,3)$ & 1,31 & $(-6,4 ; 9,1)$ & 0,804 & 0,807 \\
\hline $\operatorname{IMC}\left(\mathrm{kg} / \mathrm{m}^{2}\right)$ & 0,01 & $(-3,0 ; 3,0)$ & 0,55 & $(-2,6 ; 3,8)$ & 0,797 & 0,804 \\
\hline RCQ & 0,03 & $(-0,8 ; 0,1)$ & $-0,02$ & $(-0,6 ; 0,3)$ & 0,125 & 0,644 \\
\hline \multicolumn{7}{|l|}{ Cognitivas } \\
\hline Códigos WAIS-III & 8,68 & $(6,7 ; 10,7)$ & $-0,29$ & $(-2,4 ; 1,8)$ & $<0,001$ & $<0,001$ \\
\hline CTT-A & $-7,94$ & $(-13,1 ;-2,8)$ & $-1,75$ & $(-7,4 ; 3,9)$ & 0,016 & 0,109 \\
\hline СТT-B & $-18,59$ & $(-31,4 ;-5,8)$ & 2,25 & $(-13,4 ; 17,9)$ & 0,101 & 0,045 \\
\hline
\end{tabular}

IMC = índice de massa corporal; RCQ = relação cintura quadril; Códigos WAIS-III = Subteste Códigos da Escala Wechsler de Inteligência; CTT $=$ Color Trail Test; $\Delta$ = diferença média (Escore pós - escore pré); IC 95\% = intervalo de confiança de $95 \%$. 
(25\%) com risco moderado e $10(62,5 \%)$ com alto risco. No grupo controle, apenas uma participante foi classificada com risco moderado, enquanto que o restante apresentou alto risco. $\mathrm{Na}$ comparação das classificações de RCQ entre os grupos, não houve diferença estatisticamente significativa $(\mathrm{p}=0,140)$.

\section{Discussão}

Os resultados do presente estudo sugerem efeito da caminhada na melhora de habilidades cognitivas, enquanto que as variáveis antropométricas não apresentaram diferenças estatisticamente significativas após 12 semanas de intervenção.

Os resultados encontrados corroboram estudos prévios, os quais demonstraram que o treinamento físico melhora a função cognitiva de idosos da comunidade em situação de risco cardiovascular ${ }^{7}$, institucionalizados ${ }^{23}$, com comprometimento cognitivo leve ${ }^{24}$ e até mesmo com Alzheimer ${ }^{25}$. O diferencial do presente estudo foi a reprodução de um contexto frequentemente observado nas comunidades brasileiras, em que grupos de idosos reúnem-se matinalmente para a prática de caminhada. $\mathrm{O}$ método adotado difere-se dos modelos experimentais tradicionais que, geralmente, adotam protocolos pouco reprodutíveis à população geral e afastam-se da manifestação cultural e do contexto de vida das pessoas.

Como possíveis mecanismos de ação da atividade física na função cognitiva, existem indícios de que a mesma estimule eventos neuroprotetores, neuroplasticidade (i.e., capacidade do sistema nervoso se reorganizar, adaptando-se a novos estímulos) e melhore o estado de saúde das praticantes, beneficiando habilidades cognitivas. Nesse sentido, o exercício físico aumenta o fluxo sanguíneo cerebral, que por sua vez induz a neurogênese e a sinaptogênese $e^{26,27}$. Partindo do pressuposto de que as voluntárias não estavam habituadas à rotina de intervenção, as variações das atividades e do percurso de caminhada durante o estudo podem ter levado à formação de novos circuitos neurais, aumentando a reserva cognitiva. Além disso, a prática regular de atividade física pode ter reduzido o perfil inflamatório e o risco cardiovascular ${ }^{6,10}$, que são fatores de risco associados ao declínio cognitivo. Existe, portanto, um contexto multifatorial possivelmente associado à melhora cognitiva observada neste estudo. Ressalta-se que as ausências de biomarcadores e de neuroimagem não permitem a determinação de um mecanismo causal específico.

Houve grande tamanho de efeito na comparação da atenção e da velocidade de processamento da informa- ção (teste Códigos WAIS-III) entre os grupos, possivelmente resultante da prática de atividade física. Esse é um resultado importante, tendo em vista que o aperfeiçoamento dessas habilidades representa ganhos relevantes às interações sócioambientais. Afinal, o declínio cognitivo pode comprometer o julgamento e a tomada de decisão, fazendo com que o idoso tenha dificuldade em atividades com abundância de estímulos sensoriais, como atravessar uma rua movimentada ou dirigir durante tráfego intenso. Por isso, essas habilidades cognitivas estão diretamente associadas à segurança e à autonomia do idoso, que, quando preservadas ou aprimoradas, permitem respostas mais rápidas e assertivas em relação aos problemas circunstanciais do dia a dia.

Sobre as funções executivas, houve efeito médio e alto para os testes CTT-A e CTT-B, respectivamente. Embora o resultado indique efeito promissor da atividade física, deve-se ter parcimônia em sua generalização visto que houve grande quantidade de missings, sobretudo no teste CTT-B. Apesar de os testes serem precedidos por um período de assimilação da tarefa, é provável que o instrumento seja mais adequado em amostras de participantes mais escolarizados, parâmetro este que não foi considerado no presente estudo. Desse modo, a melhora das funções executivas indicaria maior capacidade de planejamento e execução, o que está diretamente relacionado à autonomia do idoso.

Os resultados dos presente estudo contrapõem-se às recomendações internacionais de prescrição de exercícios físicos de baixa intensidade à população idosa, nas quais sugere-se volume de treinamento de 150 a $300 \mathrm{~min} / \mathrm{semana}^{28}$. Embora essas recomendações possuam grande validade para as capacidades físicas, ainda não há consenso em relação aos efeitos nas habilidades cognitivas. No presente estudo foram encontrados resultados importantes mesmo com um volume mais baixo (120 $\mathrm{min} /$ semana).

Embora a aderência ao treinamento físico seja um desafio para profissionais de Educação Física, neste estudo houve uma experiência bem-sucedida. Existem indícios que ajudam a ententer esse resultado, como a socialização entre as voluntárias, além das sessões terem sido realizadas em ambientes abertos e arborizados, pois a prática de atividade física em parques naturais favorece à aderência e à percepção de tranquilidade ${ }^{14}$, características fundamentais à assiduidade dos praticantes. As interações promovidas pelas sessões de treinamento são capazes de estimular a formação de novas amizades e de melhorar aspectos emocionais 
como o humor e sintomas depressivos ${ }^{29}$, representando um alento ao isolamento social frequentemente observado na velhice. Houve também o estabelecimento de relações sociais que transcenderam o ambiente destinado à prática de caminhada, consistindo em um ganho social inestimável.

Sobre as variáveis antropométricas, os grupos mantiveram-se estáveis, sugerindo que o protocolo de intervenção não modificou a composição corporal analisados. Esse resultado possivelmente ocorreu em razão do não controle dietético, uma vez que em estudo anterior $^{30}, 120$ minutos semanais de treinamento aeróbio combinado com restrição calórica foram efetivos na redução da massa corporal de idosos com sobrepeso.

Como limitações do presente estudo, destacam-se: ausência de cálculo de tamanho amostral, aleatorização dos grupos, variáveis sociodemográficas, como a escolaridade, e a falta de controle da carga de treinamento e das atividades físicas realizadas fora do ambiente de intervenção. Apesar disso, essas limitações não comprometem a capacidade de generalização dos resultados e, tampouco, inviabilizam as conclusões. $\mathrm{O}$ cálculo da amostra não foi realizado por uma questão operacional, pois havia uma amostra disponível, o que é bastante frequente em investigações epidemiológicas. $\mathrm{O}$ cálculo amostral a posteriori poderia ser realizado, porém, levando em consideração que o tamanho do efeito da estimativa principal foi muito alto $(\mathrm{d}=2,182)$, o poder do teste poderia superestimar a capacidade de generalização da amostra. Sobre a ausência de aleatorização, a comparação na linha de base revelou que os grupos eram equivalentes e comparáveis, reduzindo a interferência do viés de seleção. A escolaridade e o controle da carga sinalizam direcionamentos a estudos futuros, nos quais critérios de seleção mais rígidos podem acarretar menor prevalência de missings nos testes cognitivos. Para o controle da carga, existem aplicativos de smartphone que monitoram a quantidade de passos, consistindo em um parâmetro relevante para a definição do volume. Sobre a intensidade, pode-se usar escalas de percepção subjetiva de esforço ou técnicas de monitoramento da frequência cardíaca. A adoção de biomarcadores e neuroimagem podem auxiliar o processo de estabelecimento de mecanismos causais relacionados ao desempenho cognitivo.

No presente estudo, a atividade física melhorou a atenção seletiva e a velocidade de processamento da informação de mulheres idosas destreinadas, além de apresentar resultados promissores sobre as funções exe- cutivas. Diante disso, a caminhada pode ser considerada fator de proteção contra os efeitos deletérios do envelhecimento, sobretudo à função cognitiva. Como aplicações práticas, foi apresentada uma proposta factível de ocupação do espaço público em benefício da população idosa, além da validação de práticas bastante disseminadas nas comunidades brasileiras, como a caminhada e o alongamento. Existem estratégias que podem auxiliar no maior engajamento dos praticantes, como a realização de atividades físicas em ambientes abertos e o incentivo à formação de círculos de amizade, que aumentam a motivação de cada pessoa. Evidencia-se, portanto, que a adoção de um estilo de vida mais saudável, com ações simples e efetivas, pode ajudar a manter a integridade física e psicológica no processo de envelhecimento.

\section{Conflito de interesses}

Os autores declaram não haver conflito de interesses.

\section{Contribuição dos autores}

Oliveira RA, foi o responsável pela elaboração intelectual do estudo, coleta de dados e escrita do manuscrito. Soares VN, realizou a análise estatística e participou da escrita do manuscrito. Sampaio RAC, revisou a análise estatística e o texto científico. Fernandes PT, participou de todo o processo, auxiliando na elaboração intelectual do estudo, coleta de dados e revisão crítica do manuscrito.

\section{Referências}

1. Neri AL, Yassuda MS, Araújo LFd, Eulálio MC, Cabral BE, Siqueira MEC, et al. Metodologia e perfil sociodemográfico, cognitivo e de fragilidade de idosos comunitários de sete cidades brasileiras: Estudo FIBRA. Cad Saúde Pública. 2013;29(4):778-92.

2. Holz AW, Nunes BP, Thumé E, Lange C, Facchini LA. Prevalência de déficit cognitivo e fatores associados entre idosos de Bagé, Rio Grande do Sul, Brasil. Rev Bras Epidemiol. 2013;16(4):880-8.

3. Rabelo DF. Comprometimento cognitivo leve em idosos: avaliação, fatores associados e possibilidades de intervenção. Kairós. 2009;12(2):65-79.

4. Ferreira PCS, Tavares DMS, Rodrigues RAP. Características sociodemográficas, capacidade funcional e morbidades entre idosos com e sem declínio cognitivo. Acta Paul Enferm. 2011;24(1):29-35.

5. Halil M, Kizilarslanoglu MC, Kuyumcu ME, Yesil Y, Jentoft AJC. Cognitive aspects of frailty: Mechanisms behind the link between frailty and cognitive impairment. J Nutr Health Aging. 2015;19(3):276-83.

6. Kirk-Sanchez NJ, McGough EL. Physical exercise and cognitive performance in the elderly: current perspectives. Clin Interv Aging. 2014;9:51-62.

7. Sofi F, Valecchi D, Bacci D, Abbate R, Gensini FG, Casini A, et al. Physical activity and risk of cognitive decline: a meta-analysis of prospective studies. J Intern Med. 2011;269(1):107-17. 
8. Ferreira APS, Szwarcwald CL, Damacena GN. Prevalence of obesity and associated factors in the Brazilian population: a study of data from the 2013 National Health Survey. Rev Bras Epidemiol. 2019;22:e190024.

9. Souza Barbosa JF, Santos Gomes C, Vilton Costa J, Ahmed T, Zunzunegui MV, Curcio CL, et al. Abdominal Obesity and Mobility Disability in Older Adults: A 4-Year FollowUp the International Mobility in Aging Study. J Nutr Health Aging. 2018;22(10):1228-37.

10. Casas Herrero A, Izquierdo M. Ejercicio físico como intervención eficaz en el anciano frágil. An Sist Sanit Navar. 2012;35(1):69-85.

11. Knaepen K, Goekint M, Heyman EM, Meeusen R. Neuroplasticity-exercise-induced response of peripheral brain-derived neurotrophic factor. Sports Med. 2010;40(9):765-801.

12. Chang YK, Labban JD, Gapin JI, Etnier JL. The effects of acute exercise on cognitive performance: a meta-analysis. Brain Res. 2012;1453:87-101.

13. Liu-Ambrose T, Best JR, Davis JC, Eng JJ, Lee PE, Jacova C, et al. Aerobic exercise and vascular cognitive impairment: a randomized controlled trial. Neurology. 2016;87(20):2082-90.

14. Hanson S, Jones A. Is there evidence that walking groups have health benefits? A systematic review and meta-analysis. Br J Sports Med. 2015;49(11):710-5.

15. Najas M,Yamatto TH.Avaliação do estado nutricional de idosos. Nestlé nutrition. 2008. [citado em 2019 set 19]. Disponível em: http://www.ufjf.br/renato_nunes/files/2014/03/Avalliaçãodo-estado-Nutricional-de-Idosos.pdf.

16. Lipschitz DA. Screening for nutritional status in the elderly. Prim Care. 1994;21(1):55-67.

17. Bray GA, Gray DS. Obesity. Part I--Pathogenesis. West J Med. 1988;149(4):429.

18. Nascimento E. WAIS-III: Escala de Inteligência Wechsler para Adultos-manual técnico. São Paulo: Casa do Psicólogo. 2005.

19. Rabelo IS, Pacanaro SV, Rosseti MO, Leme I. Teste de trilhas coloridas. São Paulo: Casa do Psicólogo. 2010.

20. Coutinho ACAM, Nascimento E. Formas abreviadas no WAIS-III para avaliação da inteligência. Aval Psicol. 2010;9(1):25-33.
21. Rabelo IS, Pacanaro SV, Rossetti MO, Leme IFAS, Castro NR, Güntert CM, et al. Color Trails Test: a Brazilian normative sample. Psychol Neurosci. 2010;3(1):93-9.

22. Rosenthal JA. Qualitative descriptors of strength of association and effect size. J Soc Serv Res. 1996;21(4):37-59.

23. Sanchez-Gonzalez JL, Calvo-Arenillas JI, SanchezRodriguez JL. The effects of moderate physical exercise on cognition in adults over 60 years of age. Rev Neurol. 2018;66(7):230-6.

24. Van Uffelen JGZ, Chinapaw MJM, Van Mechelen W, Hopman-Rock M. Walking or vitamin B for cognition in older adults with mild cognitive impairment? A randomized controlled trial. Br J Sports Med. 2008;42(5)2400-9.

25. Groot C, Hooghiemstra AM, Raijmakers P, Bercker BNM, Scheltens P, Scherder EJA, et al. The effect of physical activity on cognitive function in patients with dementia: A meta-analysis of randomized control trials. Ageing Res Rev. 2016;25:13-23.

26. Lista I, Sorrentino G. Biological mechanisms of physical activity in preventing cognitive decline. Cell Mol Neurobiol. 2010;30(4):493-503.

27. Draganski B, Gaser C, Busch V, Schuierer G, Bogdahn U, May A. Neuroplasticity: changes in grey matter induced by training. Nature. 2004;427(6972):311-2.

28. Thompson WR, Gordon NF, Pescatello LS, American College of Sports Medicine. ACSM's guidelines for exercise testing and prescription. 2010.

29. Barbour KA, Blumenthal JA. Exercise training and depression in older adults. Neurobiol Aging. 2005;26(1):119-23.

30. Beavers KM, Beavers DP, Martin SB, Marsh AP, Lyles MF, Lenchik L, et al. Change in bone mineral density during weight loss with resistance versus aerobic exercise training in older adults. J Gerontol A Biol Sci Med Sci. 2017;72(11):1582-5.

\section{Como citar este artigo:}

Oliveira RA, Soares VN, Sampaio RAC, Fernandes PT. Influência da caminhada na cognição e composição corporal de mulheres idosas. Rev Bras Ativ Fis Saúde. 2019;24:e0081. DOI: 10.12820/rbafs.24e0081 